\title{
Control variate method for stationary processes
}

\author{
TOMOYUKI AMANO \\ MASANOBU TANIGUCHI*
}

Department of Applied Mathematics, School of Fundamental Science and Engineering,
Waseda University, Tokyo, 169-8555, Japan

\begin{abstract}
The sample mean is one of the most natural estimators of the population mean based on independent identically distributed sample. However, if some control variate is available, it is known that the control variate method reduces the variance of the sample mean. The control variate method often assumes that the variable of intersest and the control variable are i.i.d. Here we assume that these variables are stationary processes with spectral density matrices, i.e.dependent. Then we propose an estimator of the mean of the stationary process of interest by using control variate method based on nonparametric spectral estimator. It is shown that this estimator improves the sample mean in the sense of mean square error. Also this analysis is extended to the case when the mean dynamics is of the form of regression. Then we propose a control variate estimator for the regression coefficients which improves the least squares estimator (LSE). Numerical studies will be given to see how our estimator improves the LSE.
\end{abstract}

JEL classification: C02; C10; C13; C14; C15; C22

Keywords: Control variate method; Stationary processes; Spectral density matrix; Nonparametric spectral estimator

\section{Introduction}

The sample mean is one of the most natural estimators for the population mean based on the i.i.d. sample. When some control variable vector is available (a random vector which is possibly correlated with the variable of interest), using the information about the control variate vector, it is known that the control variate method reduces the variance of the sample mean. That is, if $\bar{Y}$ is a sample mean of i.i.d.sample $\left\{Y_{i}\right\}_{i=1}^{n}$ with an unknown mean $\mu_{Y}$ and $X$ is a control variable vector with known mean vector $\mu_{X}$, then for any constant vector $b$, the mean of the control variate estimator $\hat{\mu}_{Y}(b)=\bar{Y}-b^{\prime}\left(X-\mu_{X}\right)$ for $\mu_{Y}$ is $\mu_{Y}$ and it's variance is $\operatorname{Var}[\bar{Y}]-2 b^{\prime} \operatorname{Cov}[\bar{Y}, X]+b^{\prime} \sum_{X} b$, where $\sum_{X}$ is the covariance matrix

\footnotetext{
*Corresponding author. +81-3-5286-8095.

E-mail addresses: tomtochami@ ruri.waseda.jp (T. Amano), taniguchi@waseda.jp (M.

Taniguchi)
} 
of $X$ and $\operatorname{Cov}[\bar{Y}, X]$ is the covariance vector between $\bar{Y}$ and $X$. Hence if $2 b^{\prime} \operatorname{Cov}[\bar{Y}, X]>$ $b^{\prime} \sum_{X} b$, then the variance of the control variate estimator is smaller than that of the sample mean.

This method has been discussed in the case when the sample and control variable are i.i.d.. Lavenberg and Welch (1981) reviews analyses of the control variate developed up to the date. In the paper the value $b_{*}$ of vector $b$ which minimizes the variance of the control variate estimator is derived and the confidence interval of $\hat{\mu}_{Y}\left(b_{*}\right)$ is constructed. However in practice, since the correlation between $\bar{Y}$ and $X$ is unknown, this $b_{*}$ is not known and an estimator $\hat{b}_{*}$ of $b_{*}$ is proposed. In general the control variate estimator involving the estimator $\hat{b}_{*}$ is not unbaiased and the confidence interval can not be constructed easily. They also discuss these problems. Rubinstein and Markus (1985) extends the results to the case when the sample mean $\bar{Y}$ is multidimensional vector and the multidimensional control variate estimator is represented as $\hat{\mu}_{Y}(B)=\bar{Y}-B\left(X-M_{X}\right)$, where $B$ is an arbitary matrix and $X$ is a control variate vector with mean vector $M_{X}$. They give the matrix $B_{*}$ which minimizes the determinant of $E\left\{\hat{\mu}_{Y}(B)^{\prime} \hat{\mu}_{Y}(B)\right\}$, which is called the generalized variance of $\hat{\mu}_{Y}(B)$. They also introduce an estimator of $\hat{B}_{*}$ of $B_{*}$ and discuss the confidence ellipsoid. Nelson (1990) proves a central limit theorem of the control variate estimator. Since a lot of control variate theories have been discussed under a specific probability structure (usually normal distribution) for the sample and control variates, a number of authors introduced remedies for violations of these assumptions. Nelson (1990) gives a systematic analytical evaluation of them. In recent years this method is applied to financial engineering (e.g., Glasserman (2003), Chan and Wong (2006)).

Since the control variate theory is usually discussed under the assumption that the sample and control variates are i.i.d, in this paper, when the sample is generated from a stationary process and some control variable process is available, we propose an estimator $\hat{\theta}_{C}$ of the mean of the concerned process by using control variate method. Then it is shown that this estimator improves the sample mean in the sense of mean square error (MSE). The estimator $\hat{\theta}_{C}$ is expressed in terms of nonparametric estimators for spectra of the concerned process and the control variate process. We also apply this analysis to the case when the mean dynamics is of the form of regression. A control variate estimator for the regression coefficients is proposed and is shown to improve the LSE in the sense of MSE. Numerical studies show how our estimators behave. Our results have potential application to various fields, including econometrics in particular.

This paper is organized as follows. In Section 2 we introduce an estimator $\hat{\theta}_{C}$ for the mean of a stationary process by using control variate method. Section 3 shows this estimator $\hat{\theta}_{C}$ improves the sample mean in the sense of MSE. In Section 4, control variate estimators for the mean which is of the form of regression are proposed and shown to improve the LSE. Section 5 provides numerical studies which show how our estimators improve the sample mean. Proofs of theorems are relegated to Section 6. Throughout the paper we denote the set of all integers by $\mathbf{Z}$, and denote by $\|(\cdot)\|$ the Euclidean norm of $(\cdot)$. 


\section{Setting}

One of the most fundamental estimators of the population mean is the sample mean. It is known that if the sample is i.i.d, and if some control variable is available, using the information about the control variate $X$ and its mean $\mu_{X}$, the control variate method improves the mean square error of the sample mean. In this section we apply this method to the case when the sample is generated by a stationary process and some control variate process is available, and introduce an estimator of the mean, which improves the variance of the sample mean. Suppose that $\{Y(t) ; t \in \mathbf{Z}\}$ is a scalar-valued process with mean $E[Y(t)]=\theta$ and $\{X(t) ; t \in \mathbf{Z}\}$ is an another m-dimensional process with the mean vector $E[X(t)]=0$, which is possibly correlated with $\{Y(t)\}$. We are now interested in estimation of $\theta$. Let $Z(t) \equiv\left(Y(t), X^{\prime}(t)\right)^{\prime}$. The following assumptions are imposed.

Assumption 2.1. $\{Z(t) ; t \in \mathbf{Z}\}$ is generated by the following linear process.

$$
Z(t)=\sum_{j=0}^{\infty} B(j) \epsilon(t-j)+\vec{\theta}
$$

where $\vec{\theta}=(\theta, 0,0, \cdots, 0)^{\prime}$ is $m+1$-dimensional vector and $B(j)^{\prime}$ s are $(m+1) \times(m+1)$ matrices and $\{\epsilon(t)\}$ is a sequence of i.i.d. $m+1$-dimensional random vectors with mean vector 0 and covariance matrix $K$.

Henceforth $|U|, U_{i, j}$ and $v_{i}$ denote the sum of all the absolute values of elements of matrix $U$, the $(i, j)$-th element of the matrix $U$ and the $i$-th element of vector $v$, respectively.

Assumption 2.2. (i) $\operatorname{Det}\left[\sum_{u=0}^{\infty} B(u) z^{u}\right]=0$ has no roots in the unit disc $\{z \in C ;|z| \leq 1\}$.

(ii) The coefficient matrices $B(u)$ satisfy

$$
\sum_{u=0}^{\infty}|u|^{4}|B(u)|<\infty
$$

Let $\operatorname{Cum}\left(Q_{1}, \cdots, Q_{k}\right)$ be the joint cumulant of random variables $Q_{1}, \cdots, Q_{k}$. We assume the following.

Assumption 2.3. For $k=3,4, \cdots$,

$$
C_{k}^{\epsilon} \equiv \sup _{a_{1}, \cdots, a_{k}}\left|\operatorname{Cum}\left(\epsilon_{a_{1}}(0), \cdots, \epsilon_{a_{k}}(0)\right)\right|<\infty
$$

and

$$
\sum_{L=1}^{\infty}\left(\sum_{v} C_{n_{1}}^{\epsilon} \cdots C_{n_{P}}^{\epsilon}\right) z^{L} / L !<\infty
$$


for $z$ in neighborhood of 0 , where the inner summation is over all indecomposable partitions (see Brillinger (2001), p20) $v=\left(v_{1}, \cdots, v_{P}\right)$ of the table

$$
\begin{array}{cc}
1 & 2 \\
3 & 4 \\
\vdots & \vdots \\
2 L-1 & 2 L
\end{array}
$$

with $v_{p}$ having $n_{p}>1$ elements, $p=1, \cdots, P$.

Write $\operatorname{Cum}_{a_{1}, \cdots, a_{k}}\left(t_{1}, \cdots, t_{k-1}\right)=\operatorname{Cum}\left\{Z_{a_{1}}\left(t_{1}\right), \cdots, Z_{a_{k-1}}\left(t_{k-1}\right), Z_{a_{k}}(0)\right\}$ and

$$
C_{k} \equiv \sup _{a_{1}, \cdots, a_{k}} \sum_{t_{1}, \cdots, t_{k-1}=-\infty}^{\infty}\left|\operatorname{Cum}_{a_{1}, \cdots, a_{k}}\left(t_{1}, \cdots, t_{k-1}\right)\right| .
$$

Then Assumptions 2.1, 2.2 and 2.3 imply

$$
\sum_{t_{1}, \cdots, t_{k-1}=-\infty}^{\infty}\left\{1+\left|t_{j}\right|\right\} \mid \text { Cum }_{a_{1}, \cdots, a_{k}}\left(t_{1}, \cdots, t_{k-1}\right) \mid<\infty
$$

for $j=1, \cdots, k-1$ and any $k$ tuple $a_{1}, \cdots, a_{k}$ when $k=2,3 \cdots$, and

$$
\sum_{L=1}^{\infty}\left(\sum_{v} C_{n_{1}} \cdots C_{n_{P}}\right) z^{L} / L !<\infty,
$$

where the summation $\sum_{v}$ is defined as in (2.4) (see, Brillinger (2001), p48). From Assumptions 2.1 and 2.2 , it is seen that the process $\{Z(t)\}$ becomes a stationary process with nonsingular spectral density matrix (e.g., Brillinger (2001)). We write the spectral density matrix by

$$
f(\lambda)=\left(\begin{array}{ll}
f_{Y Y}(\lambda) & f_{Y X}(\lambda) \\
f_{X Y}(\lambda) & f_{X X}(\lambda)
\end{array}\right) .
$$

From Assumption 2.2, it follows that $R(s)=\left\{\operatorname{Cum}_{i, j}(s)\right\}$ satisfies

$$
\sum_{s=-\infty}^{\infty}|s|^{4}|R(s)|<\infty
$$

(e.g., Brillinger (2001), p.46). Suppose that partial observations $\{Y(0), Y(1), \cdots Y(n-1)\}$ and $\left\{X\left(-M_{n}\right), X\left(-M_{n}+1\right), \cdots, X(0), \cdots, X(n-1)\right\}$ are available, where $M_{n}=O\left(n^{\beta}\right)$ $\left(\frac{1}{4} \leq \beta<\frac{1}{3}\right)$.

Now we are interested in the estimation of $\theta$. Based on the observations we introduce the following estimator $\hat{\theta}_{C}$ of $\theta$

$$
\hat{\theta}_{C} \equiv \frac{1}{n} \sum_{t=0}^{n-1}\left\{Y(t)-\sum_{u=0}^{M_{n}} \hat{a}_{n}^{\prime}(u) X(t-u)\right\},
$$


where $\hat{a}_{n}(u)=\frac{1}{2 \pi} \int_{-\pi}^{\pi} \hat{A}_{n}(\lambda) \exp (i u \lambda) d \lambda, \hat{A}_{n}(\lambda)=\hat{f}_{X X}(\lambda)^{-1} \hat{f}_{X Y}(\lambda)$. Here $\hat{f}_{X X}(\lambda)$ and $\hat{f}_{X Y}(\lambda)$ are, respectively, nonparametric estimators of $f_{X X}(\lambda)$ and $f_{X Y}(\lambda)$ which are defined as,

$$
\begin{aligned}
& \hat{f}_{X Y}(\lambda) \equiv \frac{2 \pi}{n} \sum_{s=1}^{n-1} W_{n}\left(\lambda-\frac{2 \pi s}{n}\right) I_{X Y}\left(\frac{2 \pi s}{n}\right) \\
& \hat{f}_{X X}(\lambda) \equiv \frac{2 \pi}{n} \sum_{s=1}^{n-1} W_{n}\left(\lambda-\frac{2 \pi s}{n}\right) I_{X X}\left(\frac{2 \pi s}{n}\right)
\end{aligned}
$$

where $I_{X Y}(\mu)$ and $I_{X X}(\mu)$ are submatrices of the periodogram

$$
\begin{aligned}
I_{n}(\mu) & \equiv \frac{1}{2 \pi n}\left\{\sum_{t=0}^{n-1} Z(t) e^{i t \mu}\right\}\left\{\sum_{t=0}^{n-1} Z(t) e^{i t \mu}\right\}^{*} \\
& =\left(\begin{array}{cc}
I_{Y Y}(\mu) & I_{Y X}(\mu) \\
I_{X Y}(\mu) & I_{X X}(\mu)
\end{array}\right) \quad(\text { say }),
\end{aligned}
$$

and $\left\{W_{n}(\lambda)\right\}$ are weight functions which are described in the next section. The $\hat{A}_{n}(\lambda)$ and $\hat{a}_{n}(u)$ are shown to be consistent estimators of $A(\lambda)=f_{X X}(\lambda)^{-1} f_{X Y}(\lambda), a(u)=$ $\frac{1}{2 \pi} \int_{-\pi}^{\pi} A(\lambda) \exp (i u \lambda) d \lambda$, respectively. In the next section we will show that the proposed estimator $\hat{\theta}_{C}$ improves the sample mean in the sense of the mean square error (MSE).

\section{Asymptotic theory}

In this section we elucidate the asymptotics of $\hat{\theta}_{C}$. Initially, we state the following assumption on $\left\{W_{n}(\lambda)\right\}$.

Assumption 3.1. $\quad$ (i)

$$
W_{n}(\lambda)=N_{n} W\left(N_{n} \lambda\right)
$$

where $N_{n}=O\left(n^{\frac{1}{3}}\right)$ and positive and $W(x)$ is bounded, even, non-negative and satisfies

$$
\int_{-\infty}^{\infty} W(x) d x=1
$$

(ii) $W_{n}(\lambda)$ can be expanded as $W_{n}(\lambda)=\frac{1}{2 \pi} \sum_{l} w\left(\frac{l}{N_{n}}\right) e^{-i l \lambda}$, where $w(x)$ is a continuous, even function with $w(0)=1,|w(x)| \leq 1$ and $\int_{-\infty}^{\infty} w(x)^{2} d x<\infty$, and satisfies $\lim _{|x| \rightarrow 0} \frac{1-w(x)}{|x|}=k_{1}<\infty$ for some constant $k_{1}$.

Then we get the following theorem.

Theorem 3.1. Suppose Assumptions 2.1, 2.2, 2.3 and 3.1. Then it holds that

$$
\lim _{n \rightarrow \infty} n E\left|\hat{\theta}_{C}-\theta\right|^{2}=2 \pi\left(f_{Y Y}(0)-f_{Y X}(0) f_{X X}(0)^{-1} f_{X Y}(0)\right) .
$$


It is known that the asymptotic variance of the sample mean $\bar{Y}_{n} \equiv \frac{1}{n} \sum_{t=0}^{n-1} Y(t)$ is $2 \pi f_{Y Y}(0)$ (e.g., Brillinger (2001), Theorem 5.2.1). Since

$$
2 \pi\left(f_{Y Y}(0)-f_{Y X}(0) f_{X X}(0)^{-1} f_{X Y}(0)\right) \leq 2 \pi f_{Y Y}(0),
$$

we observe that $\hat{\theta}_{C}$ improves $\bar{Y}_{n}$ in the sense of MSE.

Remark 3.1. If $\{X(t)\}$ has a known mean vector $\mu_{x}$, we define the control variate estimator $\hat{\theta}_{C} \equiv \frac{1}{n} \sum_{t=0}^{n-1}\left\{Y(t)-\sum_{u=0}^{M_{n}} \hat{a}_{n}^{\prime}(u)\left(X(t-u)-\mu_{x}\right)\right\}$. However if $\mu_{x}$ is unknown, we use $\tilde{\theta}_{C} \equiv \frac{1}{n} \sum_{t=0}^{n-1}\left\{Y(t)-\sum_{u=0}^{M_{n}} \hat{a}_{n}^{\prime}(u)(X(t-u)-\bar{X})\right\}$ instead of $\hat{\theta}_{C}$, where $\bar{X}=\frac{1}{n} \sum_{t=0}^{n-1} X(t)$. We mention this eligibility in Section 6.

\section{Regression models}

We assume $\{Y(t) ; t \in \mathbf{Z}\}$ is a trend model whose mean $E[Y(t)]=\mu(t)=\phi^{\prime}(t) \theta$ is a time dependent function. Here $\phi(t)=\left(\phi_{1}(t), \cdots, \phi_{J}(t)\right)^{\prime}$ and $\theta=\left(\theta_{1}, \cdots, \theta_{J}\right)^{\prime}$. Let $\{X(t) ; t \in \mathbf{Z}\}$ be an another m-dimensional process with mean vector $E[X(t)]=0$, which is possibly correlated with $\{Y(t)\}$. Now we apply the control variate method to estimate the parameter $\theta$. Let $Z(t) \equiv\left(Y(t), X^{\prime}(t)\right)^{\prime}, t \in \mathbf{Z}$. We impose the following assumption.

Assumption 4.1. $\{Z(t) ; t \in \mathbf{Z}\}$ is generated by the following linear process.

$$
Z(t)=\sum_{j=0}^{\infty} B(j) \epsilon(t-j)+\left(\begin{array}{c}
\mu(t) \\
0 \\
\vdots \\
0
\end{array}\right)
$$

where $B(j)^{\prime}$ s are $(m+1) \times(m+1)$ matrices satisfying Assumption 2.2 and $\epsilon(t)^{\prime}$ s are i.i.d. random vectors with mean vector 0 and covariance matrix $K$.

For convenience we define $\eta(t)$ by $\sum_{j=0}^{\infty} B(j) \epsilon(t-j)=\left(\eta(t), X^{\prime}(t)\right)^{\prime}$, then as discussed in Section 2., $\left(\eta(t), X^{\prime}(t)\right)^{\prime}$ has the spectral density matrix,

$$
f(\lambda)=\left(\begin{array}{ll}
f_{\eta \eta}(\lambda) & f_{\eta X}(\lambda) \\
f_{X \eta}(\lambda) & f_{X X}(\lambda)
\end{array}\right) .
$$

Suppose that partial observations $\{Y(0), Y(1), \cdots Y(n-1)\}$ and $\left\{X\left(-M_{n}\right), X\left(-M_{n}+1\right), \cdots\right.$, $X(0), \cdots, X(n-1)\}$ are available.

We define nonparametric estimators $\hat{f}_{X X}(\lambda)$ and $\hat{f}_{X \hat{\eta}}(\lambda)$ for the spectral densities $f_{X X}(\lambda)$ and $f_{X \eta}(\lambda)$, respectively, as

$$
\begin{aligned}
& \hat{f}_{X X}(\lambda) \equiv \frac{2 \pi}{n} \sum_{s=1}^{n-1} W_{n}\left(\lambda-\frac{2 \pi s}{n}\right) I_{X X}\left(\frac{2 \pi s}{n}\right) \\
& \hat{f}_{X \hat{\eta}}(\lambda) \equiv \frac{2 \pi}{n} \sum_{s=1}^{n-1} W_{n}\left(\lambda-\frac{2 \pi s}{n}\right) I_{X \hat{\eta}}\left(\frac{2 \pi s}{n}\right)
\end{aligned}
$$


where

$$
\begin{aligned}
& I_{X X}(\mu) \equiv \frac{1}{2 \pi n}\left\{\sum_{t=0}^{n-1} X(t) e^{i t \mu}\right\}\left\{\sum_{t=0}^{n-1} X(t) e^{i t \mu}\right\}^{*} \\
& I_{X \hat{\eta}}(\mu) \equiv \frac{1}{2 \pi n}\left\{\sum_{t=0}^{n-1} X(t) e^{i t \mu}\right\}\left\{\sum_{t=0}^{n-1} \hat{\eta}(t) e^{i t \mu}\right\}^{*}
\end{aligned}
$$

where $\hat{\eta}(t)=Y(t)-\phi^{\prime}(t) \bar{\theta}_{L S E}$ and $\bar{\theta}_{L S E}=\left(\phi^{\prime} \phi\right)^{-1} \phi^{\prime} Y$ (the least squares estimator of $\theta$ ). Let $\hat{A}(\lambda)=\hat{f}_{X X}(\lambda)^{-1} \hat{f}_{X \hat{\eta}}(\lambda)$ and $\hat{a}(u)=\frac{1}{2 \pi} \int_{-\pi}^{\pi} \hat{A}(\lambda) \exp (i u \lambda) d \lambda$.

Now we propose an estimator $\hat{\theta}_{L S E}^{C}$ of $\theta$ :

$$
\hat{\theta}_{L S E}^{C}=\left(\phi^{\prime} \phi\right)^{-1} \phi^{\prime}\left(Y-\hat{W}_{M}\right)
$$

where $Y=(Y(1), \cdots, Y(n))^{\prime}, \phi=(\phi(1), \cdots, \phi(n))^{\prime}$ and $\hat{W}_{M}=\left(\hat{W}_{M}(1), \cdots, \hat{W}_{M}(n)\right)^{\prime}$ with

$$
\hat{W}_{M}(t)=\sum_{u=0}^{M_{n}} \hat{a}^{\prime}(u) X(t-u) .
$$

To describe asymptotics of $\hat{\theta}_{L S E}^{C}$, we impose the following Grenander's conditions.

Assumption 4.2. Let $c_{j, k}^{n}(h)=\sum_{t=1}^{n-h} \phi_{j}(t+h) \phi_{k}(t)=\sum_{t=1-h}^{n} \phi_{j}(t+h) \phi_{k}(t) . c_{j, k}^{n}(h)$ 's satisfy the following conditions.

(i) $c_{j, j}^{n}(0)=O\left(n^{\gamma}\right), j=1, \cdots, J$ for some $\gamma>0$.

(ii) $\lim _{n \rightarrow \infty} \frac{\phi_{j}^{2}(n+1)}{c_{j, j}^{n}(0)}=0, j=1, \cdots, J$.

(iii)

$$
\lim _{n \rightarrow \infty} \frac{c_{j, k}^{n}(h)}{\left\{c_{j, j}^{n}(0) c_{k, k}^{n}(0)\right\}^{\frac{1}{2}}}=m_{j k}(h)
$$

We may take $\phi_{1}(t)=1$ (constant), which evidently satisfies Assumption 4.2, hence, the regression part $\phi(t)$ of $\{Y(t)\}$ may include a constant.

We define the $J \times J$ matrix $m_{\phi \phi}(u)$ by

$$
m_{\phi \phi}(u)=\left\{m_{j k}(u)\right\} .
$$

From Brillinger (2001, p175), there exists an $r \times r$ matrix valued function $G_{\phi \phi}(\lambda),-\pi<$ $\lambda \leq \pi$, whose entries are of bounded variation, such that

$$
m_{\phi \phi}(u)=\int_{-\pi}^{\pi} \exp (i u \lambda) d G_{\phi \phi}(\lambda)
$$

for $u=0, \pm 1, \cdots$. Under these assumptions, we obtain the following theorem. 
Theorem 4.1. Suppose Assumptions 2.3, 3.1, 4.1, 4.2, then

$$
\lim _{n \rightarrow \infty} n^{\gamma} E\left[\left(\hat{\theta}_{L S E}^{C}-\theta\right)\left(\hat{\theta}_{L S E}^{C}-\theta\right)^{\prime}\right]=2 \pi m_{\phi \phi}(0)^{-1} \int_{-\pi}^{\pi} f_{\eta-V, \eta-V}(\lambda) d G_{\phi \phi}(\lambda) m_{\phi \phi}(0)^{-1},
$$

where $f_{\eta-V, \eta-V}(\lambda)=f_{\eta, \eta}(\lambda)-f_{\eta X}(\lambda) f_{X X}(\lambda)^{-1} f_{X \eta}(\lambda)$ is the spectral density of $\eta(t)-V(t)$. Here $V(t)=\sum_{u=0}^{\infty} a^{\prime}(u) X(t-u), \quad a(u)=\frac{1}{2 \pi} \int_{-\pi}^{\pi} A(\lambda) \exp (i u \lambda) d \lambda, \quad A(\lambda)=f_{X X}(\lambda)^{-1} f_{X \eta}(\lambda)$.

Note that the least squares estimator $\bar{\theta}_{L S E}$ of $\theta$ has the following asymptotic variance

$$
\lim _{n \rightarrow \infty} n^{\gamma} E\left[\left(\bar{\theta}_{L S E}-\theta\right)\left(\bar{\theta}_{L S E}-\theta\right)^{\prime}\right]=2 \pi m_{\phi \phi}(0)^{-1} \int_{-\pi}^{\pi} f_{\eta, \eta}(\lambda) d G_{\phi \phi}(\lambda) m_{\phi \phi}(0)^{-1},
$$

where $f_{\eta, \eta}(\lambda)$ is the spectral density of $\eta(t)$. It is seen that

$$
f_{\eta-v, \eta-v}(\lambda) \equiv f_{\eta, \eta}(\lambda)-f_{\eta X}(\lambda) f_{X X}(\lambda)^{-1} f_{X \eta}(\lambda) \leq f_{\eta, \eta}(\lambda),
$$

which implies that the asymptotic covariance matrix of $\hat{\theta}_{L S E}^{C}$ is smaller than that of $\bar{\theta}_{L S E}$.

\section{Numerical study}

In this section we examine our control variate estimators numerically. By simulation, we compare the control variate estimators with sample means in Example 5.1 and with the least squares estimators in Example 5.2. Example 5.3 deals with real financial data. Then we see how our estimator improves the sample mean and least squares estimator.

Example 5.1. Let us consider the following process of interest $\{Y(t)\}$ and control process $\{X(t)\}$

$$
\begin{aligned}
& Y(t)=u(t)+v(t) \\
& X(t)=a_{1} u(t)+0.4 u(t-1)+a_{2} v(t)
\end{aligned}
$$

where $a_{1}, a_{2}$ are constant values. Here $\{u(t)\}$ and $\{v(t)\}$ are mutually independent, and $\{u(t)\},\{v(t)\}$ are i.i.d. $N(0,1)$. Based on 1000 observations for $\{Y(t)\}$ and $\{X(t)\}$, first, in the setting of (2.1), we evaluate the sample mean square error (SMSE) of the control variate estimator $\hat{\theta}_{C}$ and the sample mean $\bar{Y}$. In what follows we set $M_{n}=20$.

In Table 1, we report the SMSE of $\hat{\theta}_{C}$ and $\bar{Y}$ for the various values of $a_{1}, a_{2}$ by 50 times simulation.

\begin{tabular}{|cc||lc|c|}
\hline$a_{1}$ & $a_{2}$ & \multicolumn{1}{|c|}{ SMSE $\hat{\theta}_{C}$} & \multicolumn{1}{c|}{ SMSE $\bar{Y}$} & SMSE $\bar{Y}-$ SMSE $\hat{\theta}_{C}$ \\
\hline \hline 1.0 & 0.2 & 0.00047551 & 0.001357404 & 0.000881894 \\
0.8 & 0.4 & 0.000255565 & 0.001616194 & 0.001360629 \\
0.6 & 0.6 & 0.000141104 & 0.001955541 & 0.001814437 \\
0.4 & 0.8 & 0.000000364 & 0.002562325 & 0.002561961 \\
0.2 & 1.0 & 0.000098874 & 0.003079185 & 0.002980311 \\
0.2 & 2.0 & 0.000344068 & 0.001222061 & 0.000877993 \\
\hline
\end{tabular}


Table 1: The SMSE of $\hat{\theta}_{C}$ and $\bar{Y}$

From Table 1, we can see SMSE $\bar{Y}-\mathrm{SMSE} \hat{\theta}_{C}$ becomes larger when the coefficient $a_{2}$ of error process $v(t)$ becomes large, which implies, if control variates are highly correlated with the disturbance, then $\hat{\theta}_{C}$ is better than $\bar{Y}$. However excessive influence of the disturbance makes the performance of the control variate estimator worse.

Example 5.2. Let us consider the following process of interest $\{Y(t)\}$ and control process $\{X(t)\}$

$$
\begin{aligned}
& Y(t)=\mu(t)+u(t)+v(t) \\
& X(t)=0.5 u(t)+0.4 u(t-1)+a_{1} v(t)
\end{aligned}
$$

where $a_{1}$ is a constant value, $\mu(t)=\theta^{\prime} \phi(t), \phi(t)$ is a regression function and $\theta$ is a vector valued parameter. Here $\{u(t)\}$ and $\{v(t)\}$ are mutually independent, and $\{u(t)\},\{v(t)\}$ are i.i.d. $N(0,1)$. The sample sizes of $Y(t)$ and $X(t)$ are 1000 , respectively. For these samples the control variate estimator $\hat{\theta}_{L S E}^{C}$ and the least squares estimator $\bar{\theta}_{L S E}^{C}$ are calculated. We repeat this procedure 50 times (write $i$-th control variate estimator and least squares estimator as $\hat{\theta}_{L S E}^{(i) C}$ and $\bar{\theta}_{L S E}^{(i)}$ ) and evaluate $S M S E_{C} \equiv \frac{1}{50} \sum_{i=1}^{50}\left\|\hat{\theta}_{L S E}^{(i) C}-\theta\right\|^{2}$ and $S M S E_{L S E} \equiv$ $\frac{1}{50} \sum_{i=1}^{50}\left\|\bar{\theta}_{L S E}^{(i) C}-\theta\right\|^{2}$ for the regression function $\phi(t)=(1, t)^{\prime}$ and $\phi(t)=\left(1, \cos \left(\frac{\pi}{4} t\right)\right)^{\prime}$. The true value of $\theta=\left(\theta_{1}, \theta_{2}\right)^{\prime}$ is assumed to be $(1,1)^{\prime}$.

For various values of $a_{1}$ we evaluate the $\mathrm{SMSE}_{C}$ and $\operatorname{SMSE}_{L S E}$ for $\phi(t)=(1, t)^{\prime}$ in Table 2 and for $\phi(t)=\left(1, \cos \left(\frac{\pi}{4} t\right)\right)^{\prime}$ in Table 3, respectively.

\begin{tabular}{|c||cc|c|}
\hline$a_{1}$ & $\mathrm{SMSE}_{C}$ & $\mathrm{SMSE}_{L S E}$ & $\mathrm{SMSE}_{L S E}-\mathrm{SMSE}_{C}$ \\
\hline \hline 0.3 & 0.000000264 & 0.000086314 & 0.00008605 \\
0.5 & 0.000008198 & 0.000595965 & 0.000587767 \\
0.7 & 0.000061842 & 0.005833247 & 0.005771405 \\
\hline
\end{tabular}

Table 2: $\operatorname{SMSE}_{C}$ and $\operatorname{SMSE}_{L S E}\left(\phi(t)=(1, t)^{\prime}\right)$

\begin{tabular}{|c||cc|c|}
\hline$a_{1}$ & $\mathrm{SMSE}_{C}$ & $\mathrm{SMSE}_{L S E}$ & $\mathrm{SMSE}_{L S E}-\mathrm{SMSE}_{C}$ \\
\hline \hline 0.3 & 0.000197033 & 0.003661844 & 0.003464811 \\
0.5 & 0.000638327 & 0.004169522 & 0.003531195 \\
0.7 & 0.000059859 & 0.017065923 & 0.017006064 \\
\hline
\end{tabular}

Table 3: $\operatorname{SMSE}_{C}$ and $\operatorname{SMSE}_{L S E}\left(\phi(t)=\left(1, \cos \left(\frac{\pi}{4} t\right)\right)^{\prime}\right)$

From Tables 2 and 3, $\operatorname{SMSE}_{C}$ is smaller than $\operatorname{SMSE}_{L S E}$ as the coefficient $a_{1}$ of error process increases. That is, highly correlated control variates with disturbance make $\hat{\theta}_{L S E}^{C}$ better than $\bar{\theta}_{L S E}$. The next example deals with real financial data.

Example 5.3. We calculate the control variate estimator $\hat{\theta}_{C}$ and the sample mean $\bar{Y}$ of NIPPON OIL CORPORATION's log return of stock price $\{Y(t)\}$ from 7/19/2007 to 12/12/2007 by setting the difference between Yen-Euro's exchange rate and its sample 
mean from 7/4/2007 to 12/11/2007 as the control variate process. Then, using the control variate estimator $\hat{\theta}_{C}$ and the sample mean $\bar{Y}$, we forecast NIPPON OIL CORPORATION's stock $S(N)$ at $N=12 / 13 / 2007$, that is, the estimators for $S(N)$ are calculated by $\hat{S}^{C}(N) \equiv e^{\hat{\theta}_{C}+\log S(N-1)}$ and $\hat{S}(N) \equiv e^{\bar{Y}+\log S(N-1)}$.

The results are given in Table 4.

\begin{tabular}{|ccc|}
\hline$\hat{S}^{C}(N)$ & $\hat{S}(N)$ & $S(N)$ \\
\hline \hline 891.0968 & 891.4768 & 891 \\
\hline
\end{tabular}

Table 4.

From Table 4, the prediction value $\hat{S}^{C}(N)$ is nearer to the true value $S(N)$ than $\hat{S}(N)$, which implies the prediction by the control variate estimator is better than that by the sample mean.

There are many fields (econometrics, natural sciences, medical sciences etc.) where we should identify the statistical models for data of interest under the circumstance that we can use some related variables. In such situations, our estimators $\hat{\theta}_{C}$ and $\hat{\theta}_{L S E}^{C}$ can be applied, and are more efficient than the usual estimators.

\section{Acknowledgements}

The authors would like to thank for referees for their comments, which improved the original version of this paper.

\section{REFERENCES}

[1] Brillinger, D. R., 2001, Time Series Data Analysis and Theory. Society for Industrial and Applied Mathematics.

[2] Chan, N. H., Wong, H. Y., 2006, Simulation techniques in financial risk management. Wiley, New York.

[3] Fuller, W. A., 1996, Introduction to statistical time series. Wiley, New York.

[4] Glasserman, P., 2004, Monte Carlo methods in financial engineering. Springer, New York.

[5] Lavenberg, S. S., Welch, P. D., 1981, A perspective on the use of control variables to increase the efficiency of Monte Carlo simulations. Management Sci. 27, 322-335.

[6] Magnus, J. R., Neudecker, H., 1999, Matrix differential calculus with applications in statistics and econometrics. Wiley, New York.

[7] Nelson, B. L., 1990, Control variate remedies. Oper. Res. 38, 974-992.

[8] Rubinstein, R. Y., Marcus, R., 1985, Efficiency of multivariate control variates in Monte Carlo simulation. Oper. Res. 33, 661-677. 